\title{
Clinical Risk Factors and Plaque Characteristics Associated with New Development of Contralateral Stenosis in Patients Undergoing Carotid Endarterectomy
}

\author{
Sophie Merckelbach ${ }^{a}$ Tesse Leunissen ${ }^{a, b}$ Joyce Vrijenhoek ${ }^{a, c, d}$ Frans Moll ${ }^{a}$ \\ Gerard Pasterkamp $^{b, c}$ Gert Jan de Borst ${ }^{a}$

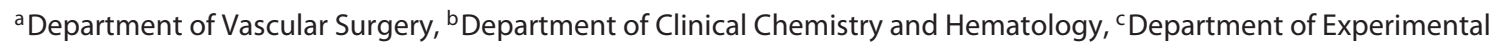 \\ Cardiology, University Medical Center Utrecht, and ${ }^{\mathrm{d}}$ Interuniversity Cardiology Institute of the Netherlands, \\ Utrecht, The Netherlands
}

\section{Key Words \\ Carotid endarterectomy $\cdot$ Carotid revascularization . \\ Contralateral stenosis · Plaque characteristics $\cdot$ Stenosis progression · Follow-up}

\begin{abstract}
Background: Following carotid endarterectomy (CEA), cerebrovascular hemodynamic may be hampered by ipsilateral restenosis or development of contralateral stenosis. It remains to be clarified if these patients need follow-up for identifying development of contralateral stenosis. Identification of risk factors contributing to development of contralateral stenosis could allow more specific follow-up. In this current study, we assessed clinical risk factors and plaque characteristics of patients undergoing CEA with development of new contralateral stenosis during mid-term followup. Methods: Seven hundred and sixty patients undergoing CEA between 2003 and 2011 at UMC Utrecht were included. Atherosclerotic plaques were excised and analyzed for smooth muscle cells (SMCs), collagen, macrophages, lipid core, plaque hemorrhage and vessel density. Patients underwent clinical and duplex ultrasound follow-up at 3 and 12 months and yearly thereafter. Association between plaque-
\end{abstract}

\begin{tabular}{ll}
\hline KARGER & $\begin{array}{l}\text { ( } 2016 \text { The Author(s) } \\
\text { Published by S. Karger AG, Basel } \\
\text { 1015-9770/16/0422-0122\$39.50/0 }\end{array}$ \\
E-Mail karger@karger.com & This article is licensed under the Creative Commons Attribution- \\
www.karger.com/ced & $\begin{array}{l}\text { NonCommercial-NoDerivatives 4.0 International License (CC BY- } \\
\text { NC-ND) (http://www.karger.com/Services/OpenAccessLicense). } \\
\text { Usage and distribution for commercial purposes as well as any dis- } \\
\text { tribution of modified material requires written permission. }\end{array}$
\end{tabular}

Tesse Leunissen

Department of Vascular Surgery

University Medical Center Utrech

Heidelberglaan 100, NL-3584 CX Utrecht (The Netherlands)

E-Mail T.C.Leunissen@ umcutrecht.nl ral stenosis $\geq 50 \%$ was assessed with univariate and multivariate analysis. Clinical outcome during follow-up was associated with development of new contralateral stenosis. Results: After a median follow-up time of 2.5 years, development of contralateral stenosis was observed in 108 patients $(20 \%)$. Presence of high collagen $(p=0.025)$ and high SMC $(p=0.027)$ was associated with development of new contralateral stenosis, whereas large lipid core was negatively associated with new development of contralateral stenosis $(p=0.034)$. The same plaque characteristics were related to contralateral occlusion. History of coronary artery disease $(p=0.031)$ and asymptomatic presentation $(p=0.000)$ were univariably associated with development of contralateral stenosis. Multiple regression analysis indicated that asymptomatic status was independently associated with contralateral stenosis $(p=0.001)$. Patients with new development of contralateral stenosis more often showed symptoms during follow-up ( $p=0.049)$. Conclusion: Dissection of a lipid-poor, collagen-rich or SMC-rich plaque yielded an association with development of new contralateral stenosis during mid-term follow-up after CEA. Asymptomatic patients had a significantly higher risk for development of contralateral stenosis. New contralateral stenosis was related to the presence of tribution of modified material requires written permission. 
new cerebral symptoms. These findings may help to develop individual treatment algorithms for patients with cerebrovascular atherosclerotic burden. @ 2016 The Author(s)

Published by S. Karger AG, Basel

\section{Introduction}

Carotid endarterectomy (CEA) reduces the risk of stroke for both symptomatic and subgroups of asymptomatic patients with high-grade carotid artery stenosis [1]. Nevertheless, this advantage may be attenuated by the development of restenosis. Restenosis is associated with a higher risk of recurrent symptoms, re-interventions and stroke and therefore hampers the benefit of CEA [2]. In addition, following CEA patients are prone to develop atherosclerosis at other sites. Development of contralateral stenosis is relatively common after CEA, exceeds the rate of ipsilateral restenosis, reduces the contralateral cerebrovascular reserve and may also affect clinical outcome [3-7]. It remains to be clarified if patients at risk for development of contralateral stenosis need follow-up and, if so, for what period of time. Risk factors that might contribute to the development of contralateral stenosis remain to be established $[1,8]$. Identifying patients at risk for developing contralateral stenosis would allow selective individualized follow-up after CEA. Next to clinical and demographic factors, it could be useful to establish which plaque characteristics are predictive for contralateral stenosis development. Potentially, information derived from the removed carotid atherosclerotic plaque individualizes treatment decisions by using non-invasive plaque imaging [9].

Our objectives were to establish the incidence and risk factors - both clinically and based on plaque characteristics - for development of new contralateral stenosis or occlusion in mid-term follow-up after CEA.

\section{Methods}

\section{Patient Population}

All patients included in this study are part of the Athero-Express study. The design of this study has been described earlier [10]. In summary, this is a longitudinal bio bank study comprising carotid plaques of patients undergoing a primary CEA and longterm follow-up. The plaques are collected and subjected to histological examination. All patients undergoing CEA at the University Medical Center Utrecht (UMCU), The Netherlands, were asked to participate. For the present study, we examined available data from all carotid arteries operated in patients included in the Athero-Express biobank from June 2003 until September 2011.
There were no exclusion criteria. Indications for CEA were reviewed by a multidisciplinary vascular team and were based on recommendations of the Asymptomatic Carotid Atherosclerosis Study and Asymptomatic Carotid Surgery Trial studies for asymptomatic patients and the North American Symptomatic Carotid Endarterectomy Trial and European Carotid Surgery Trial studies for symptomatic patients $[3,11,12]$. This study was approved by the Institutional Review boards of the UMCU hospital. All patients provided informed consent. At baseline, patients completed a questionnaire regarding medication use, cardiovascular risk factors and medical history.

\section{Atherosclerotic Plaque Examination}

CEA was performed by experienced vascular surgeons. Method of arterial closure was chosen by the surgeon and could be either primary or patch closure with Dacron, bovine or vein patch. The procedure remained the same during the inclusion period. The atherosclerotic plaque was immediately brought to the laboratory after removal during CEA. Plaque characteristics were scored by 2 independent observers blinded for clinical outcome with a good intra-observer and inter-observer reproducibility [9]. Scoring was analyzed in 2 categories (no/minor or moderate/heavy staining) for macrophages, smooth muscle cells (SMCs), calcification, collagen and lipid core. Plaque hemorrhage and fibrin staining were defined as absent or present. Vessel density was binned in 2 groups (low and high), split by the median of this density. More extensive information about tissue collection, tissue processing and histological examination is described in earlier articles $[13,14]$.

\section{Duplex Follow-Up}

A follow-up with duplex ultrasound took place at 3 and 12 months after CEA and yearly thereafter. An IU22 ultrasound device from Philips Medical Systems (Eindhoven, The Netherlands) with linear transducer 9-3 MHz was used. The technicians who performed the duplexes were blinded for any data concerning this study. All the data regarding the duplex measurements were collected retrospectively and any lost to follow-up were defined as missing data on all follow-up moments thereafter.

\section{Clinical Outcome}

Clinical follow-up of patients was performed using annual questionnaires, from inclusion date until lost to follow-up (or latest September 2013). Records of the patients in the electronic hospital database were reviewed for cardiovascular end points. Stroke (both ipsilateral and contralateral) and death were recorded for this study. Outcomes were verified by 2 independent researchers.

\section{Definition of Endpoints}

The primary end point of follow-up in this study was the new development of contralateral stenosis at any time point. Contralateral stenosis is defined as occurrence of stenosis $\geq 50 \%$ or occlusion. In our database, stenosis was classified in the following categories, adopted from the MRC European Carotid Surgery Trial: no stenosis $(0-29 \%)$, mild stenosis $(30-49 \%)$, moderate stenosis (50-69\%), severe stenosis (70-99\%) and occlusion $[3,15,16]$. This corresponds with the following peak systolic values (PSV): no stenosis $(150 \mathrm{~cm} / \mathrm{s})$, mild stenosis $(150>$ PSV $>210 \mathrm{~cm} / \mathrm{s})$, moderate stenosis $(210>$ PSV $>270 \mathrm{~cm} / \mathrm{s}$ ), severe stenosis (PSV $>270 \mathrm{~cm} / \mathrm{s}$ ), subtotal occlusion (PSV $<40 \mathrm{~cm} / \mathrm{s}$ and severe plaque) and occlusion (no flow). 


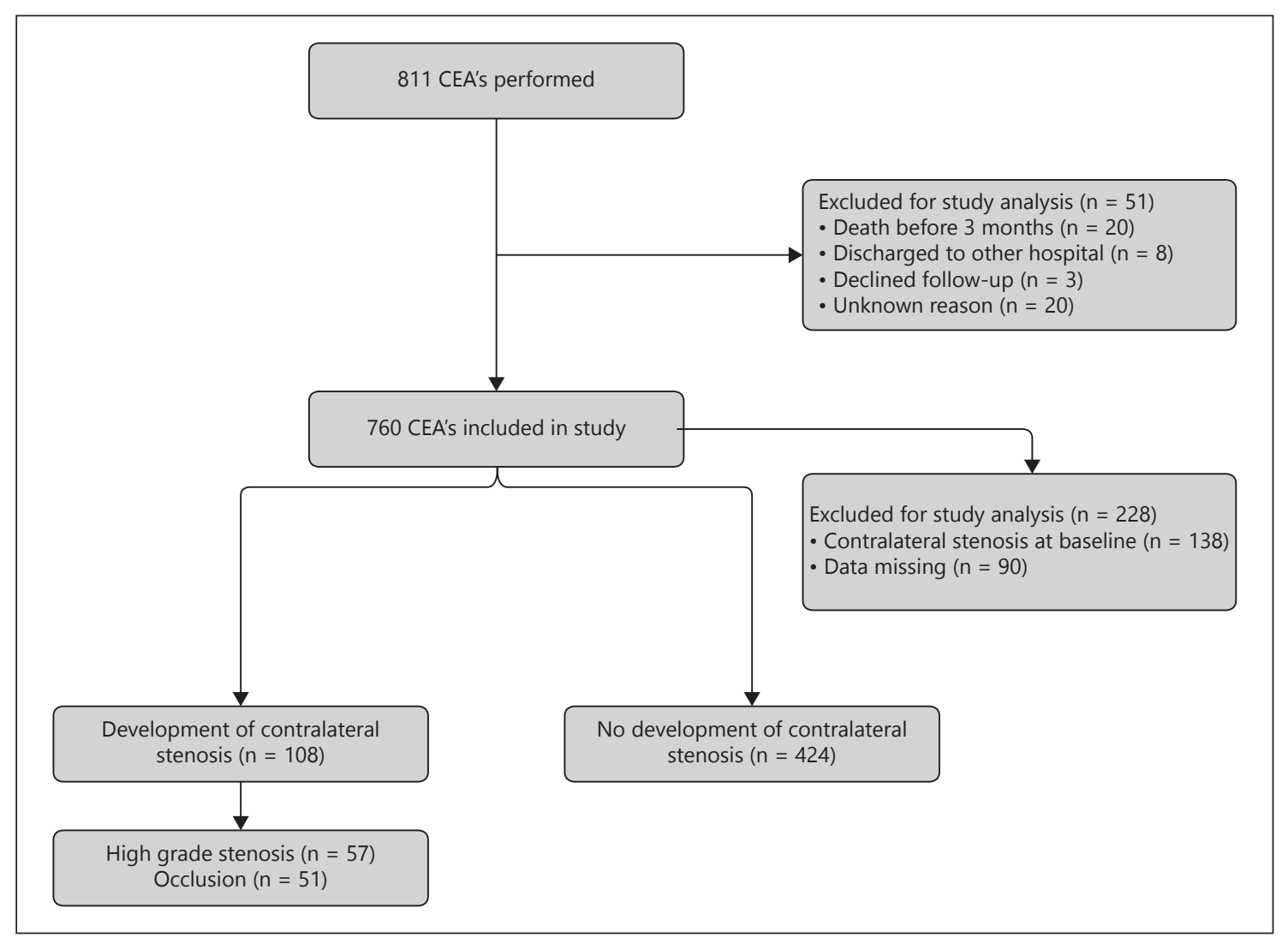

Fig. 1. Flowchart.

\section{Data Analysis}

SPSS version 20.0 (SPSS Inc., Chicago, Ill., USA) was used for all statistical analyses. Cross tables $(2 \times 2)$ were constructed to calculate the percentage of the development of contralateral stenosis for each baseline characteristic. The p value was calculated with the chi-square statistic. Continuous variables were analyzed using independent samples t tests. To adjust for potential confounders, we performed a multivariable logistic regression analysis. We assumed a potential association for the clinical risk factors sex, age, hypertension, current smoking, diabetes, body mass index, acetylsalicylic acid and statin use, symptom status, type of patch used and year of operation $[14,17]$. Additionally, a factor was assumed to be a potential confounder if a $p$ value of $<0.25$ was found in univariate analysis with development of contralateral stenosis as outcome. The survival free rate of development of contralateral stenosis was analyzed using Kaplan-Meier curves.

\section{Results}

From 2003 until 2011, in total 811 primary CEAs were performed, of which 760 patients were included in this study. In total, 51 patients were excluded because no follow-up was available (flowchart; fig. 1).
The mean age of the included patients was 69 years and $30 \%$ were women. At the moment of inclusion, smoking was reported in $33 \%$, diabetes mellitus in $23 \%$ and hypertension in $74 \%$. Clinical presentation was in $87 \%$ of the patients symptomatic, mostly comprising a transient ischemic attack (TIA). A majority of the patients used preoperative acetylsalicylic acid (82\%) and statins (82\%). Bilateral stenosis was found in $34 \%$ (138 of 401) of the patients, since they revealed a contralateral carotid stenosis of $>50 \%$ at baseline DUS. Appendix 1 comprises the full baseline characteristics of all included patients.

\section{Clinical and Plaque Characteristics after Follow-Up}

The median follow-up of the total cohort was 2.5 years (IQR 1.7-4.3). During this follow-up, 108 (20.3\%) patients developed a new contralateral carotid stenosis of more than $50 \%$. In 51 of these patients occlusion was detected. Of the total cohort, 424 patients did not develop contralateral stenosis, 138 were excluded because of contralateral stenosis at baseline and data were missing in 90 patients. In the flowchart, those numbers are stated more clearly. Patients with a history of coronary artery disease 
Table 1. Characteristics of patients with and without contralateral stenosis or occlusion in follow-up

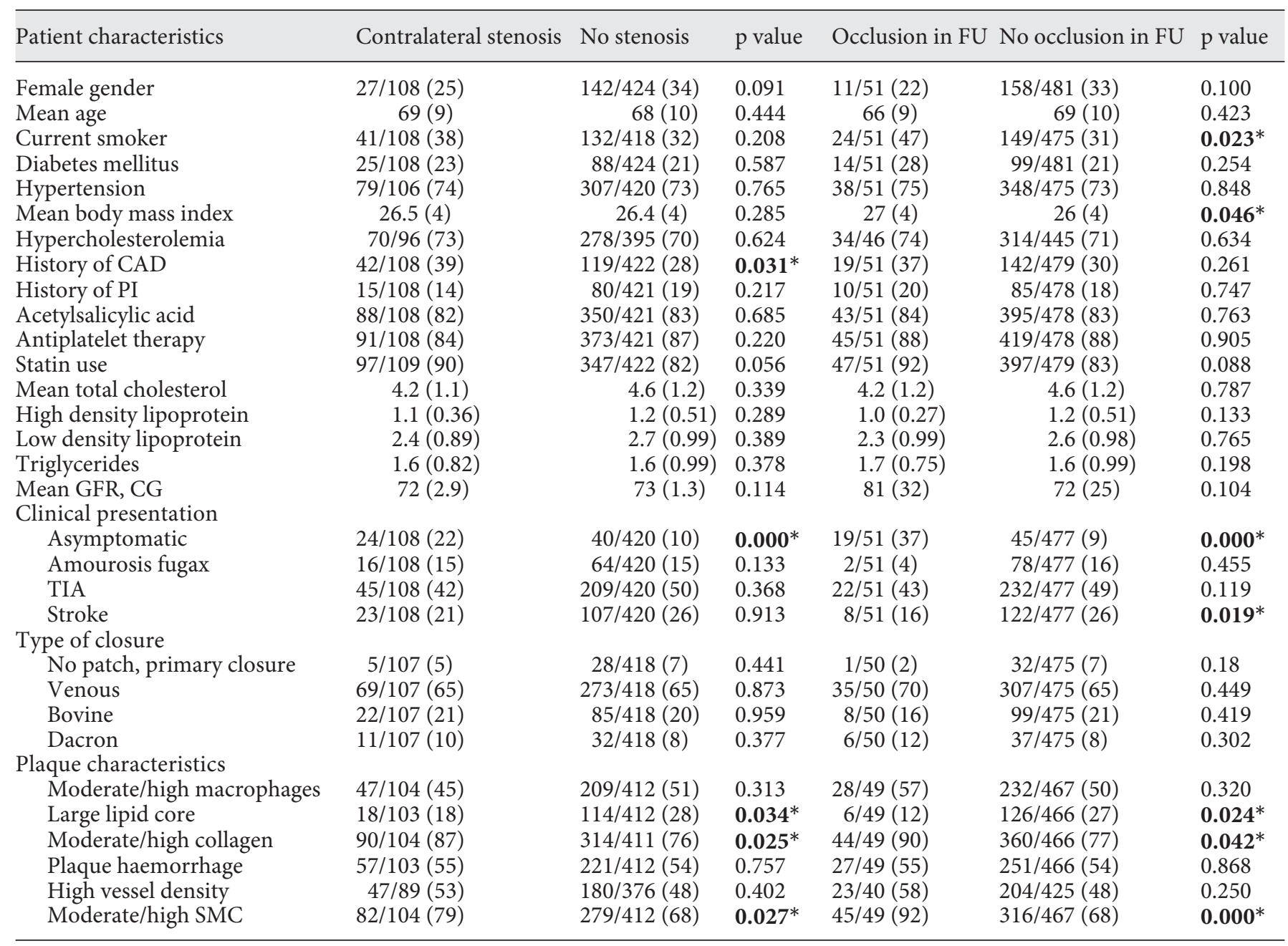

Numbers in bold $=* \mathrm{p}<0.05$. Continuous values are expressed as mean with SD, unless specified otherwise. Categorical values are expressed as n/total (\%). PI = Peripheral intervention; GFR = glomerular filtration rate; CG = Cockcroft-Gault.

(CAD) more often showed development of contralateral stenosis ( $p=0.031$; table 1$)$. Development of contralateral stenosis was also found significantly more often in patients who were asymptomatic at time of inclusion compared to those who were symptomatic (with TIA, amaurosis fugax or stroke). Other patient characteristics did not show a significant association with the development of contralateral stenosis in our study population. Patients with an atherosclerotic plaque with a large lipid core showed significantly less development of contralateral stenosis $(18 \%$ of the patients with development of contralateral stenosis had a large lipid core vs. $28 \%$ of the patients with no stenosis, $\mathrm{p}=0.034)$. High collagen and high SMC in the plaque were associated with more devel- opment of contralateral stenosis $(\mathrm{p}=0.025$ and $\mathrm{p}=0.027)$. Other plaque characteristics showed no correlation with development of contralateral stenosis in follow-up, which is shown in figure 2 .

As previously mentioned, in half of the patients with new contralateral atherosclerotic development, a contralateral occlusion developed. Predicting clinical risk factors for development of a new contralateral occlusion were current smoking ( $47 \%$ in group with occlusion vs. $31 \%$ in group without, $\mathrm{p}=0.023)$ and body mass index $(\mathrm{p}=0.046)$. Besides that, asymptomatic patients significantly more often developed contralateral occlusion during follow-up $(\mathrm{p}=0.000)$. Same plaque characteristics that were predictable for the development of contralateral stenosis were as- 


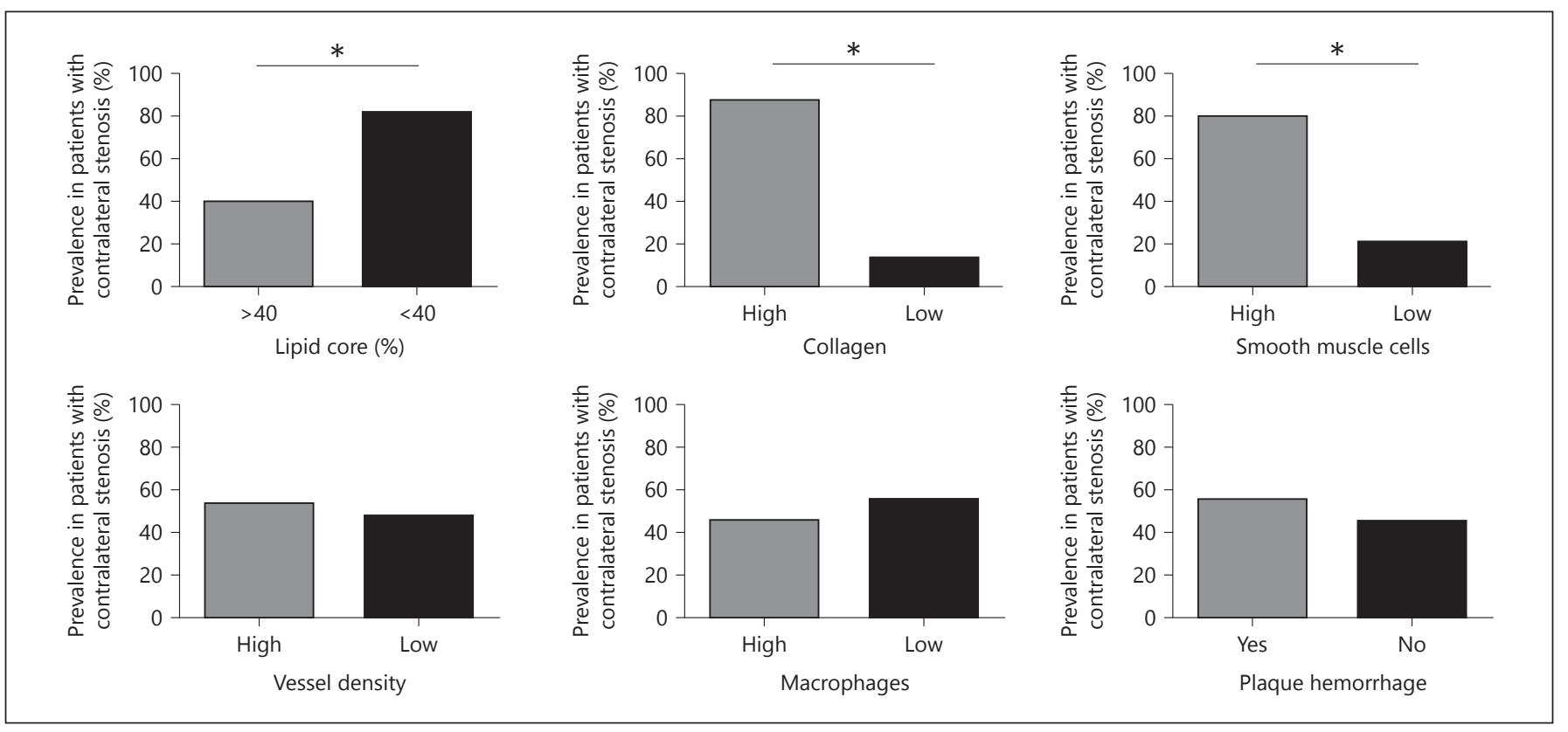

Fig. 2. Contralateral stenosis at mid-term follow-up by different plaque characteristics.

Table 2. Multi-regression analysis of potential cofounders

\begin{tabular}{ll}
\hline Patient characteristics & Significance, p value \\
\hline Female gender & 0.311 \\
Mean age & 0.163 \\
Current smoker & 0.080 \\
Diabetes mellitus & 0.507 \\
Hypertension & 0.888 \\
Mean body mass index & 0.675 \\
History of CAD & 0.014 \\
History of PI & 0.135 \\
Acetylsalicylic acid & 0.255 \\
Statin & 0.090 \\
Mean GFR, CG & 0.871 \\
Year of operation & 0.930 \\
Clinical presentation & \\
$\quad$ Asymptomatic & $0.001^{*}$ \\
$\quad$ Stroke & 0.455 \\
Type of closure & \\
$\quad$ No patch, primary closure & 0.508 \\
Bovine & 0.704 \\
Dacron & 0.383 \\
Plaque characteristics & \\
Large lipid core & 0.628 \\
Moderate/high collagen & 0.650 \\
Moderate/high SMC & 0.628 \\
\hline Adjusted R-square & 0.040 \\
F-test significance & 0.009 \\
\hline
\end{tabular}

${ }^{*}$ p value $<0.01$ shows significance. PI $=$ Peripheral intervention; GFR = glomerular filtration rate; CG = Cockcroft-Gault. sociated with the development of contralateral occlusion. In table 1 , clinical risk factors and plaque-characteristics for both patient groups with and without development of contralateral stenosis and occlusion are reported.

A multi-regression analysis to estimate the relation between development of contralateral stenosis and potential factors mentioned earlier plus the potential confounders found in univariate analysis, was performed until only variables with $p$ value $<0.01$ in the model were left. The potential confounders found in univariate analysis were history of CAD, history of peripheral intervention, glomerular filtration rate, large lipid core, high collagen and high SMC. After this analysis, only asymptomatic status before CEA turned out to be associated with development of contralateral stenosis $(p=0.001)$, values for significance are showed in table 2.

\section{Progression of Baseline Stenosis}

One hundred and thirty-eight patients showed a contralateral stenosis at baseline and were therefore excluded from the initial study analysis, in which we only looked at new development of contralateral stenosis. Of these 138 patients, $9(34.6 \%)$ patients with a baseline moderate contralateral stenosis progressed during follow-up into a severe stenosis. Eight (14.5\%) patients with baseline severe contralateral stenosis developed a (sub)total occlusion. Other patients already displayed a (sub)total occlusion at baseline. No plaque characteristics were correlated with 
Fig. 3. Kaplan-Meier curve for contralateral stenosis-free survival rate.

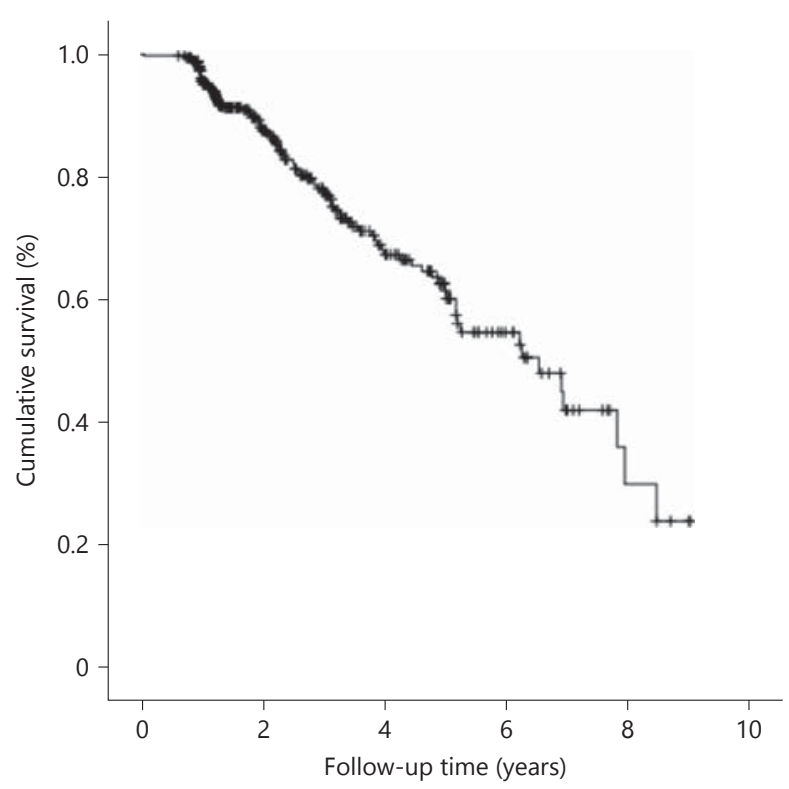

progression of contralateral stenosis except for the presence of a high amount of SMCs for patients with 50-69\% stenosis at baseline who progressed into a higher category during follow-up $(\mathrm{p}=0.046)$.

\section{Clinical Outcome}

Clinical outcome was scored by looking at symptoms of TIA, stroke or amaurosis fugax that the patients showed during follow-up. Patients who developed new contralateral stenosis during follow-up showed significantly more often symptoms than those who did not developed a contralateral stenosis (14.3. vs. $6.6 \%, \mathrm{p}=0.049$ ). $17.4 \%$ of the patients with contralateral stenosis at baseline showed symptoms during follow-up.

During follow-up, $25.3 \%$ of the patients with baseline contralateral stenosis and $6.1 \%$ of patients with new developed contralateral stenosis underwent contralateral CEA.

\section{Survival Rates}

Life table analysis showed a contralateral stenosis-free survival rate of $97 \%(\mathrm{n}=471), 89 \%(\mathrm{n}=408)$ and $79 \%(\mathrm{n}=$ 202), respectively, at 1, 2 and 3 years of follow-up. After 8 years of follow-up, 34\% of the patients did not have contralateral stenosis $(\mathrm{n}=12)$. Kaplan-Meier survival analysis showed the same survival rates (fig. 3). The greatest risk for development of contralateral stenosis was between 6 and 9 years of follow-up (hazard ratio 0.24) and median survival free time was 6 years. When patients were grouped as asymptomatic and symptomatic at inclusion, life table analysis demonstrated that asymptomatic patients display earlier and more often development of contralateral stenosis. The hazards ratio was 0.27 after 3 years in asymptomatic patients, and 0.26 after 6 years in symptomatic patients.

Cumulative stroke-free survival for all included patients after 3 years of follow-up was $93 \%$ and after 9 years $59 \%$, with a median follow-up time of 8.2 years ( $95 \%$ CI $7.8-8.5$ years). Overall survival was $91 \%$ after both 3 and 9 years, with and median follow-up time of 8.4 (95\% CI 8.1-8.6 years).

\section{Discussion}

In this study, we aimed to explore the relation of clinical risk factors and plaque characteristics with the new development of contralateral stenosis in the follow-up after CEA. We reported new development of contralateral stenosis after CEA in 1 of every 5 patients. This finding corresponds with findings in other studies $[8,18,19]$. New development of contralateral stenosis appeared after a median follow-up time of 2.5 years and approximately half of the developed stenosis was an occlusion.

Further analysis showed that patients with a history of CAD are more likely to develop contralateral stenosis in follow-up. Patients who were asymptomatic at inclusion showed a significant correlation with the development of contralateral stenosis and occlusion. The association of asymptomatic presentation and new development of ste- 
nosis has previously been described for ipsilateral restenosis $[14,17]$.

Next, we showed that a small lipid core is significantly associated with new development of contralateral stenosis. Previous studies have reported a correlation between small lipid core and development of ipsilateral restenosis after CEA $[9,10]$. The results of our study could therefore underline that atherosclerosis is a generated disease of the vascular system $[20,21]$, by showing risk factors for development of stenosis of the carotid artery that are similar to risk factors for ipsilateral stenosis, as found in previous studies. However, preceding studies of our research group could not find any association between large lipid core in local plaques and the occurrence of systemic cardiovascular outcomes. In contrast, local plaque hemorrhage was shown to be the single independent predictor of outcome [13].

In this study, we found that plaques with high collagen or high SMCs were associated with more development of contralateral stenosis. Especially, SMCs are components of a stable plaque phenotype, characterized by less inflammation and lower lipid content [22]. Earlier studies reported that removal of a stable atherosclerotic plaque gives more restenosis 1 year after follow-up $[9,14]$. In our study, removal of a stable plaque gives more contralateral stenosis, which suggests again that vascular disease is more extensive than only in the operated artery.

Furthermore, previous studies showed that lesions in asymptomatic patients have a more stable atherosclerotic plaque composition with smaller lipid cores. Symptomatic patients, who have had a TIA or stroke, show more unstable carotid plaques, with high macrophage contents and larger lipid cores [10]. In line with these studies, we found that asymptomatic patients more often had a stable plaque with a small lipid core and high SMC and also developed more often contralateral stenosis (data not shown). Summarizing, dissection of a stable atherosclerotic plaque with small lipid core and high content of SMCs increase the risk of new contralateral stenosis, as previously described for the development of ipsilateral restenosis.

In this study, the grade of stenosis was measured using duplex ultrasound-derived PSV values. It could therefore be possible that restenosis in the ipsilateral artery also leads to higher PSV values in the contralateral artery and is thus measured as contralateral stenosis [23]. However, in half the cases of development of contralateral stenosis, an occlusion is reported, which is measured as no PSV flow. Therefore, our findings cannot completely be declared with this assumption.

Finally, based on the results of this study, it can be considered to include asymptomatic patients and patients with a stable atherosclerotic plaque in a more intensive and long-term follow-up scheme. It would be useful to construct a prediction model that predicts the risk for restenosis and contralateral stenosis for each individual patient, since we showed that risk factors for contralateral stenosis are comparable to risk factors for ipsilateral restenosis indicated in earlier studies. This would allow more tailored treatment and selective follow-up.

Our study has several limitations. First, duplex ultrasound scanning is a technique with drawbacks. Measurements can vary between different types of equipment, laboratories and observers. Equipment and laboratory have not changed during this study, but different technicians have performed the measurements. This may result in intra-observer variability; however, we expect that this influence is negligible. Second, we defined contralateral stenosis as $>50 \%$ with a PSV $>210 \mathrm{~cm} / \mathrm{s}$, but cutoff values for a stenosis grade differ across studies and vascular laboratories [14]. This could lead to outcomes that would be assessed differently somewhere else.

\section{Conclusion}

One in 5 patients develops new contralateral stenosis after CEA. Asymptomatic patients prior to CEA had a higher risk for developing contralateral stenosis. An atherosclerotic plaque removed during CEA with a small lipid core, a high amount of SMCs and/or a high amount of collagen increases the risk of new development of contralateral stenosis after CEA. New contralateral stenosis was related to the presence of new cerebral symptoms. Our study results may help to develop individual treatment algorithms based on clinical presentation and plaque characteristics for patients undergoing CEA.

\section{Acknowledgments}

The authors would like to thank Evelyn Velema and Sander van de Weg for their (continuing) technical and administrative work for the Athero-Express study.

\section{Funding}

The authors received no special funding for this work.

\section{Disclosure Statement}

None of the authors have a conflict of interest to declare. 


\section{Appendix 1}

Baseline characteristics of the study population

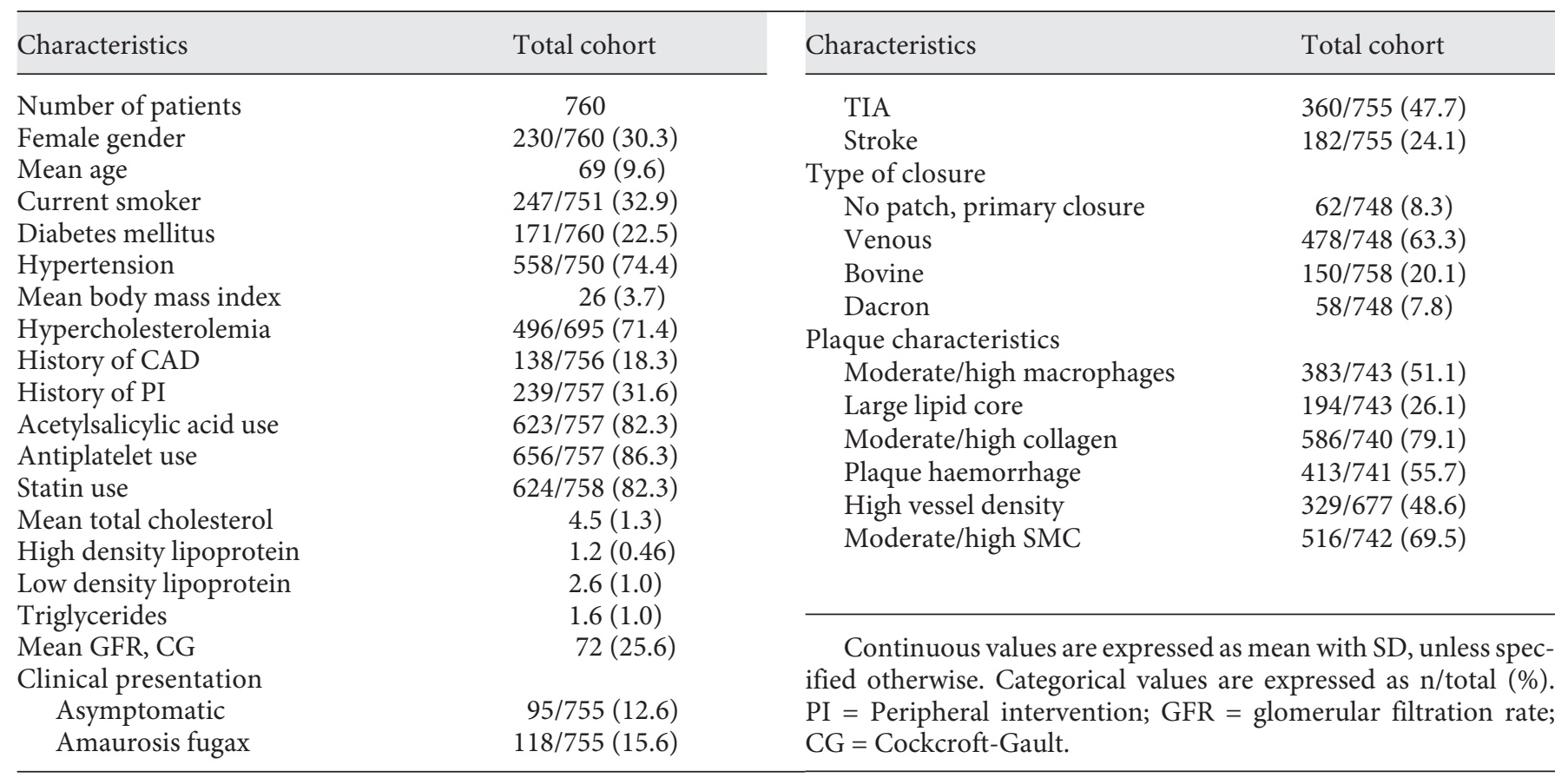

\section{References}

1 Reina-Gutiérrez T, Serrano-Hernando FJ, Sánchez-Hervás L, Ponce A, Vega de Ceniga M, Martín A: Recurrent carotid artery stenosis following endarterectomy: natural history and risk factors. Eur J Vasc Endovasc Surg 2005;29:334-341.

-2 Frericks H, Kievit J, van Baalen JM, van Bockel JH: Carotid recurrent stenosis and risk of ipsilateral stroke: a systematic review of the literature. Stroke 1998;29:244-250.

-3 Randomised trial of endarterectomy for recently symptomatic carotid stenosis: final results of the MRC European Carotid Surgery Trial (ECST). Lancet 1998;351:13791387.

-4 Fluri F, Engelter ST, Wasner M, Stierli P, Merlo A, Lyrer PA: The probability of restenosis, contralateral disease progression, and late neurologic events following carotid endarterectomy: a long-term follow-up study. Cerebrovasc Dis 2008;26: 654-658.

5 Martin-Conejero A, Reina-Gutierrez T, Serrano-Hernando FJ, Sanchez-Hervas L, Blanco-Canibano E, Ponce-Cano AI, Morata-Barrado PC, Zudaire-Diaz Tejeiro R: Disease progression in the contralateral carotid artery after endarterectomy. Ann Vasc Surg 2005; 19:662-668.
6 Raman KG, Layne S, Makaroun MS, Kelley ME, Rhee RY, Tzeng E, Muluk VS, Muluk SC: Disease progression in contralateral carotid artery is common after endarterectomy. J Vasc Surg 2004;39:52-57.

7 Sam K, Small E, Poublanc J, Han JS, Mandell DM, Fisher JA, Crawley AP, Mikulis DJ: Reduced contralateral cerebrovascular reserve in patients with unilateral steno-occlusive disease. Cerebrovasc Dis 2014;38:94100.

> Iafrati MD, Salamipour H, Young C, Mackey WC, O'Donnell TF Jr: Who needs surveillance of the contralateral carotid artery? Am J Surg 1996;172:136-139.

-9 Hellings WE, Moll FL, De Vries JP, Ackerstaff RG, Seldenrijk KA, Met R, Velema E, Derksen WJ, De Kleijn DP, Pasterkamp G: Atherosclerotic plaque composition and occurrence of restenosis after carotid endarterectomy. JAMA 2008;299:547-554.

10 Verhoeven B, Hellings WE, Moll FL, de Vries JP, de Kleijn DP, de Bruin P, Busser E, Schoneveld AH, Pasterkamp G: Carotid atherosclerotic plaques in patients with transient ischemic attacks and stroke have unstable characteristics compared with plaques in asymptomatic and amaurosis fugax patients. J Vasc Surg 2005;42:1075-1081.
11 Ferguson GG, Eliasziw M, Barr HW, Clagett GP, Barnes RW, Wallace MC, Taylor DW, Haynes RB, Finan JW, Hachinski VC, Barnett HJ: The North American Symptomatic Carotid Endarterectomy Trial: surgical results in 1415 patients. Stroke 1999;30:17511758.

12 Mayberg MR, Wilson SE, Yatsu F, Weiss DG, Messina L, Hershey LA, Colling C, Eskridge J, Deykin D, Winn HR: Carotid endarterectomy and prevention of cerebral ischemia in symptomatic carotid stenosis. Veterans Affairs Cooperative Studies Program 309 Trialist Group. JAMA 1991;266:3289-3294.

13 Hellings WE, Peeters W, Moll FL, Piers SR, van Setten J, Van der Spek PJ, de Vries JP, Seldenrijk KA, De Bruin PC, Vink A, Velema E, de Kleijn DP, Pasterkamp G: Composition of carotid atherosclerotic plaque is associated with cardiovascular outcome: a prognostic study. Circulation 2010;121: 1941-1950.

14 Vrijenhoek JE, de Borst GJ, den Ruijter HM, Merckelbach SM, de Kleijn DP, Pasterkamp G, Moll FL: A lipid-poor plaque and asymptomatic status in women are associated with higher peak systolic velocity on duplex ultrasound after carotid endarterectomy. Atherosclerosis 2014;237:677-683. 
15 Rothwell PM, Eliasziw M, Gutnikov SA, Fox AJ, Taylor DW, Mayberg MR, Warlow CP, Barnett HJ; Carotid Endarterectomy Trialists' Collaboration: Analysis of pooled data from the randomised controlled trials of endarterectomy for symptomatic carotid stenosis. Lancet 2003;361:107-116.

16 MRC European Carotid Surgery Trial: interim results for symptomatic patients with severe (70-99\%) or with mild (0-29\%) carotid stenosis. European Carotid Surgery Trialists' Collaborative Group. Lancet 1991;337:12351243.
17 van Lammeren GW, Peeters W, de Vries JP, de Kleijn DP, De Borst GJ, Pasterkamp G, Moll FL: Restenosis after carotid surgery: the importance of clinical presentation and preoperative timing. Stroke 2011;42:965-971.

18 AbuRahma AF, Cook CC, Metz MJ, Wulu JT Jr, Bartolucci A: Natural history of carotid artery stenosis contralateral to endarterectomy: results from two randomized prospective trials. J Vasc Surg 2003;38:1154-1161.

19 Ballotta E, Da Giau G, Meneghetti G, Barbon B, Militello C, Baracchini C: Progression of atherosclerosis in asymptomatic carotid arteries after contralateral endarterectomy: a 10 -year prospective study. J Vasc Surg 2007; 45:516-522.

20 Arora R, Rai F: Atherothrombosis and the management of the vulnerable vascular patient. Am J Ther 2009;16:24-34.
1 Drouet L: Atherothrombosis as a systemic disease. Cerebrovasc Dis 2002;13(suppl 1):1-6.

22 Davies MJ, Richardson PD, Woolf N, Katz DR, Mann J: Risk of thrombosis in human atherosclerotic plaques: role of extracellular lipid, macrophage, and smooth muscle cell content. Br Heart J 1993;69:377-381.

23 AbuRahma AF, Richmond BK, Robinson PA, Khan S, Pollack JA, Alberts S: Effect of contralateral severe stenosis or carotid occlusion on duplex criteria of ipsilateral stenoses: comparative study of various duplex parameters. J Vasc Surg 1995;22:751-761; discussion 761762. 